\title{
Assay based on electrical impedance spectroscopy to discriminate between normal and cancerous mammalian cells
}

\author{
Fabián Eduardo Giana, ${ }^{1,2,3,{ }^{*}}$ Fabián José Bonetto, ${ }^{1,2,3}$ and Mariela Inés Bellotti ${ }^{1, \dagger}$ \\ ${ }^{1}$ Laboratorio de Cavitación y Biotecnología, Centro Atómico Bariloche, Río Negro, R8402AGP, Argentina \\ ${ }^{2}$ Instituto Balseiro, Universidad Nacional de Cuyo/Comisión Nacional de Energía Atómica, Bariloche, Río Negro, R8402AGP, Argentina \\ ${ }^{3}$ Consejo Nacional de Investigaciones Científicas y Técnicas (CONICET), Buenos Aires, C1033AAJ, Argentina
}

(Received 24 August 2017; revised manuscript received 29 January 2018; published 19 March 2018)

\begin{abstract}
In this work we present an assay to discriminate between normal and cancerous cells. The method is based on the measurement of electrical impedance spectra of in vitro cell cultures. We developed a protocol consisting on four consecutive measurement phases, each of them designed to obtain different information about the cell cultures. Through the analysis of the measured data, 26 characteristic features were obtained for both cell types. From the complete set of features, we selected the most relevant in terms of their discriminant capacity by means of conventional statistical tests. A linear discriminant analysis was then carried out on the selected features, allowing the classification of the samples in normal or cancerous with $4.5 \%$ of false positives and no false negatives.
\end{abstract}

DOI: 10.1103/PhysRevE.97.032410

\section{INTRODUCTION}

Cancer diagnosis is a process through which a series of tests is performed to confirm that a patient actually has cancer [1]. This means that a diagnosis is carried out only when there is information indicating the possibility of suffering cancer. This kind of information is obtained normally before the appearance of symptoms though a screening test [2] or, in the worst case, when the patient shows symptoms related to some form of cancer. Depending on the location of the suspected tissue and the characteristics of the patient, there are several diagnosis procedures [1]:

(1) Laboratory tests carried out on blood samples and other body fluids: Higher or lower levels of some substance (with respect to average values found in the medical literature) could indicate the presence of cancer.

(2) Imaging techniques, from which the most used are computerized tomography (CT) and positron emission tomography (PET). The former provides anatomical information while the latter analyzes the biochemical activity, and they are generally combined in the context of cancer diagnosis.

(3) Analysis of biopsies: This procedure involves the removal of a tissue sample and its subsequent examination by a pathologist. Biopsies are normally necessary to achieve a definitive diagnosis.

Some of the mentioned procedures (principally the laboratory tests) involve the measurement of a numerical value corresponding to a biological parameter (e.g., the number of white blood cells per cubic millimeter in a blood sample). These kinds of parameters are known as indicators, because their values (compared to normal values obtained through medical research in a vast number of patients) indicate whether the body is functioning properly or if there is some variation

\footnotetext{
*fabian.giana@ib.edu.ar

†bellotti@cab.cnea.gov.ar
}

that could be explained by a disease affecting the patient. Indicators are fundamental tools for the diagnosis of cancer, and they are always used in combination with other procedures to achieve a diagnosis.

The reliability of a cancer diagnosis method is determined mainly by its sensitivity (probability that the result of the test will be positive for a patient that has cancer) and specificity (probability that a test result will be negative for a patient without cancer and positive for one with it). Both measures are combined in the so-called rates of false positives and false negatives [1]. A false positive occurs when a result that should be negative is reported as positive, and the opposite occurs in a false negative. For example, in a previous investigation involving a total of 70 patients, bronchoscopy-based lung cancer diagnosis presented $18.2 \%$ of false positives and $16.2 \%$ of false negatives, while the same diagnosis carried out using computerized tomography yielded $36.4 \%$ of false positives and $18.9 \%$ of false negatives [3]. Screening tests are also evaluated by this measure of reliability. For example, a broad study of breast cancer screening by means of digital mammography carried out on 405191 women gave as result $9.4 \%$ of false positives and $0.11 \%$ of false negatives [4].

It is of interest to develop an automatic assay to discriminate between normal and cancerous cells to obtain an indicator which could be used on cancer diagnosis. The experimental technique known as electric cell-substrate impedance sensing (ECIS, also impedance spectroscopy) has proven to be very sensitive and versatile in evaluating morphological and functional properties of cell cultures and has found many different applications over the last three decades, concerning the study of cell migration and proliferation, toxicity, wound-healing, and so on. One of the first experiments carried out with this technique involved the analysis of spectral impedance of normal and cancerous cells [5]. In that work, differences between the in vitro electrical responses of both cell types were found and the technique came out as a possible means to achieve a new discrimination method. However, these differences were not 
deeply analyzed in posterior works and the results were used to demonstrate the utility of the ECIS technique as a means to study different aspects of in vitro cell cultures.

Impedance spectroscopy is nowadays an established research tool and it has already been used in many studies involving cancer research. Most of these works aimed to comprehend different aspects concerning the development of the disease and also to evaluate new potential treatments [6-10]. However, only a few of them evaluated the possibility of using impedance measurements to discriminate between normal and cancerous cells. One of the first studies carried out in this context involved in vivo impedance measurements of normal and cancerous tissues, and the results showed some contrast between them which was attributed to the differences in the cell membrane capacity and water content [11]. Cancer diagnosis methods have also been proposed based on in vivo impedance measurements $[12,13]$, but the results were inconclusive and a clear discrimination between tumors and normal tissues could not be achieved. Afterwards, an in vivo skin cancer detector was proposed based solely on a linear regression analysis of the impedance magnitudes and phases measured on apparent lesions and surrounding healthy tissues [14]. More recently, Lovelady et al. showed that short-time fluctuations present in the impedance signals of confluent cell monolayers have different properties for normal and cancerous cultures [15]. All the aforementioned studies were based on the comparison between impedance spectra of confluent cancerous and normal cultures. Nevertheless, to our knowledge, a systematic and unified methodology has not yet been reported which allows an unknown sample to be classified as being normal or cancerous by means of a combined analysis of different spectral impedance measurement techniques.

In this work we present an assay based on impedance spectroscopy that aims to obtain relevant information which could be used to predict whether an unknown cell culture behaves as normal or cancerous. The measurements were divided in four phases according to the state of development of the cell layers. Different characteristic parameters, which are known as features, were obtained in each phase. The resulting set of features was first reduced by eliminating the less relevant, i.e., those showing low discrimination capacity. Finally, a linear discriminant analysis was performed on the set of remaining features and all the information given by the impedance measurements was combined into one single real number which could be used as an indicator on cancer diagnosis. The results indicate that the method is reliable in discriminating between normal NMuMG and cancerous LM3 cells.

\section{MATERIALS AND METHODS}

\section{A. Cell cultures}

We employed the normal cell line NMuMG (ATCC, CRL1636) [16] and the cancerous line LM3 [17]. NMuMG is an epithelial cell line from normal glandular tissue of mice, while LM3 is a murine mammary adenocarcinoma cell line. Before culturing the cells, a pre-treatment step was carried out in which $200 \mu \mathrm{l}$ of a $10 \mathrm{mM}$ solution of L-cysteine in water were added to each well and the system was left 15 min at room temperature to improve experiment repeatability [18]. The electrodes were afterwards incubated with media for approximately $24 \mathrm{~h}$ before seeding the cells, to allow proteins to pretreat the electrode surface, enhancing the attachment of cells. The culture medium was the same for both cell lines and was composed of 57\% Dulbecco's modified Eagle's medium (DMEM F-12, Gibco), 30\% modified Eagle's medium (MEM, Gibco), $10 \%$ fetal bovine serum, $1 \%$ nonessential amino acids, and $2 \%$ HEPES buffer. The final $\mathrm{pH}$ of the medium was 7.4. Cells were cultured by standard procedures described in a previous work [19] at a density of approximately $1 \times 10^{6}$ cells $\mathrm{ml}^{-1}$. We used cell suspensions with passage numbers ranging from 7 to 12 and 8 to 14 for NMuMG and LM3 cells, respectively. The culture medium on the wells was changed every $12 \mathrm{~h}$, pausing the measurements for a total of $30 \mathrm{~min}$ to allow for temperature stabilization.

\section{B. Measurement protocol}

Theoretically, cell discrimination could be achieved by comparing a set of features that characterize each cell type as a different population. Because we are working with impedance spectroscopy, the choice of these features should be based on prior knowledge about the electrical behavior of both cell lines. The information that can be obtained through impedance spectroscopy depends on the type of measurements carried out and the state of the cell layer being analyzed. The sequence of states that follow the inoculation of cells on a naked (i.e., cell-free) electrode can be summarized as follows:

(1) First, the system's impedance increases as the cells attach and spread until completely covering the electrode's surface. We call this transient interval "growth phase." It is known that cancerous cells tend to proliferate more extensively than normal cells [5], and these differences should be reflected in the dynamics of the growth phase. Based on prior results (not presented in this work), we designed this measurement to have a duration of $18 \mathrm{~h}$, to capture the whole dynamics of the process.

(2) When the cells have finally covered the available surface, they form a confluent layer and the system's impedance shows only slight, short-time fluctuations. We call this stationary state "confluent phase." Here, spectral impedance measurements give relevant information that reflects biological aspects of the cells, as explained more deeply in Sec. III B. The noiselike signals that can be obtained during micromotion have been thoroughly analyzed in a previous work [15] and we did not include them in our analysis.

(3) During the confluent phase, cells can be deliberately damaged to carry out a wound-healing assay. This kind of assay, when performed properly, can give information about the dynamics of the transient regime that corresponds to the cell death process [20] in what we call the "wounding phase."

(4) Finally, after the cell layer has been intensely damaged, the healthy cells that remain on the culture well tend to reproduce and migrate to repopulate the damaged area, increasing the impedance of the system. We expected both cell types to behave differently during this "healing phase" because of the uncontrolled growth of cancerous cells.

Figure 1 summarizes the measurement protocol developed and used in this work. 


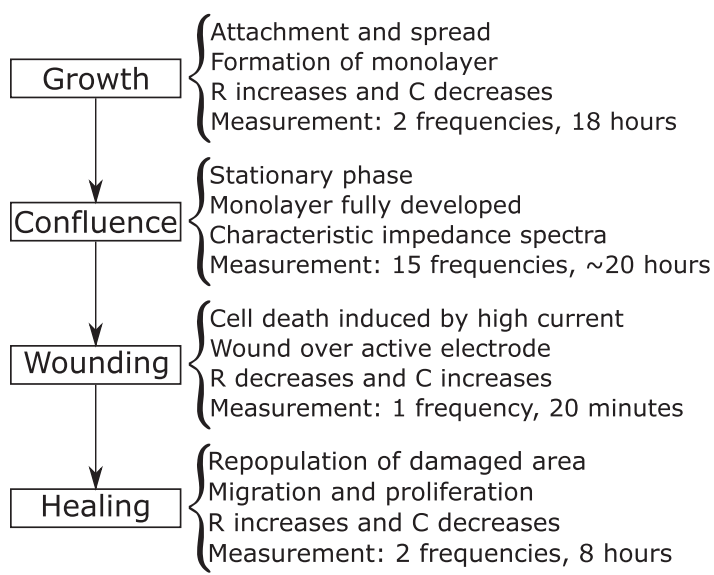

FIG. 1. Measurement protocol. Each phase is characterized by a specific behavior of the cell culture and different measurement schemes are adopted in each one. During the growth phase, impedance was registered as a function of time at two different frequencies, which were selected according to the sensitivity of the measurement (Section III A). In the confluence phase, impedance was measured at 15 different frequencies between $20 \mathrm{~Hz}$ and $100 \mathrm{kHz}$, covering the relevant part of the spectrum (Sec. IIIB). During the wounding phase, impedance was measured as a function of time at the frequency of the wounding signal, namely $30 \mathrm{kHz}$ (Sec. IIIC). Finally, during the healing phase impedance was registered as a function of time at the same frequencies used in the growth phase (Sec. IIID).

\section{Measurement schemes}

\section{Noninvasive measurements}

All noninvasive impedance measurements carried out in this paper were performed by following a typical impedance spectroscopy scheme as reported in a previous work [19] and a diagram is shown in Fig. 2. A sinusoidal waveform of $100 \mathrm{mV}_{\mathrm{RMS}}$ (Hewlett-Packard, HP33120A Function Generator) was applied to the active electrode through a $100 \mathrm{k} \Omega$ series resistor, to produce noninvasive alternating currents with amplitudes less than $1 \mu \mathrm{A}_{\mathrm{RMS}}$. The in-phase and out-ofphase voltages were measured by means of a phase-sensitive lock-in amplifier (Stanford Research Systems, SR530), setting the post demodulator low-pass filter time constant to $1 \mathrm{~s}$. These quantities were converted to real and imaginary parts of the system's impedance, which are presented formally as resistance and capacitance of a series-equivalent RC circuit [21]. The different active electrodes were selected by means of a custom-made digital demultiplexing circuit based on the CD4051BE chip (Texas Instruments), whose AC performance characteristics are adequate for these measurements. A personal computer with custom-made software was used to control the experiment. We employed commercial electrodes (Applied BioPhysics) with an active area of $5 \times 10^{-4} \mathrm{~cm}^{2}$. This setup was used to measure the naked and cell-covered electrodes during the growth, confluence and healing phases. Spectral measurements were carried out at 15 logarithmically equally spaced frequencies ranging from $20 \mathrm{~Hz}$ to $100 \mathrm{kHz}$.

\section{Cell wounding measurements}

Typical wound-healing assays performed on confluent cell monolayers are aimed to monitor cell migration and prolifera-

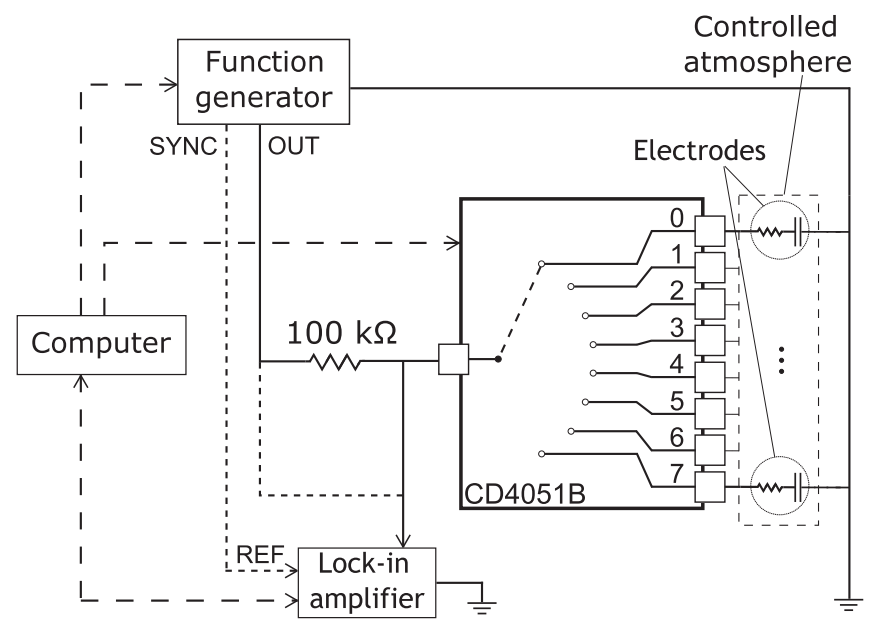

FIG. 2. Noninvasive measurement scheme adopted in this work. The function generator applies a $100 \mathrm{mV}_{\mathrm{RMS}}$ sinusoidal signal to the electrodes through a $100 \mathrm{k} \Omega$ series resistor. The lock-in amplifier measures the magnitude and phase angle of the voltage drop across the electrodes, which are modeled as series-equivalent RC circuits. The computer controls the measurement parameters, selects the active electrode to be measured through the demultiplexing circuit and processes the data.

tion after a defined portion of the layer has been mechanically or electrically damaged [22-25]. A different approach has been used in a previous work to study the dynamics of the wounding phase [20], i.e., during the time at which the wound develops. We performed an analogous assay to analyze impedance changes during the wounding phase, with some modifications. We used an LCR-meter (Agilent Technologies, E4980a) connected to the electrodes through the same demultiplexing circuit as described in Fig. 2 and without the series resistor. The amplitudes of the wounding and control signals were $2 \mathrm{~V}_{\mathrm{RMS}}$ and $20 \mathrm{mV}_{\mathrm{RMS}}$, respectively, and the frequency was in both cases $30 \mathrm{kHz}$. A wounding cycle consisted of an alternation between high- and low-voltage measurements until the electrode was exposed to the wounding signal for $60 \mathrm{~s}$. Then, the next electrode was selected via the demultiplexing circuit and a new wounding cycle was started. This procedure was repeated until completing a total of 20 cycles for each electrode, giving an effective wounding time of $20 \mathrm{~min}$. The purpose of this cascadelike approach was to have all electrodes in approximately the same condition before starting the healing phase.

\section{EXPERIMENTAL RESULTS}

\section{A. Growth phase}

An impedance spectrum of each cell-free electrode was measured right before seeding the cells on the culture wells. Figures 3(a) and 3(b) show the time variation of the normalized resistance (i.e., the measured resistance at each time divided by the naked electrode's resistance) and capacitance, respectively, for eight wells during the growth phase in a typical experiment. Note that both the normalized resistance and capacitance are dimensionless quantities. Three of these wells were seeded with NMuMG cells and three with LM3 cells, while the other 

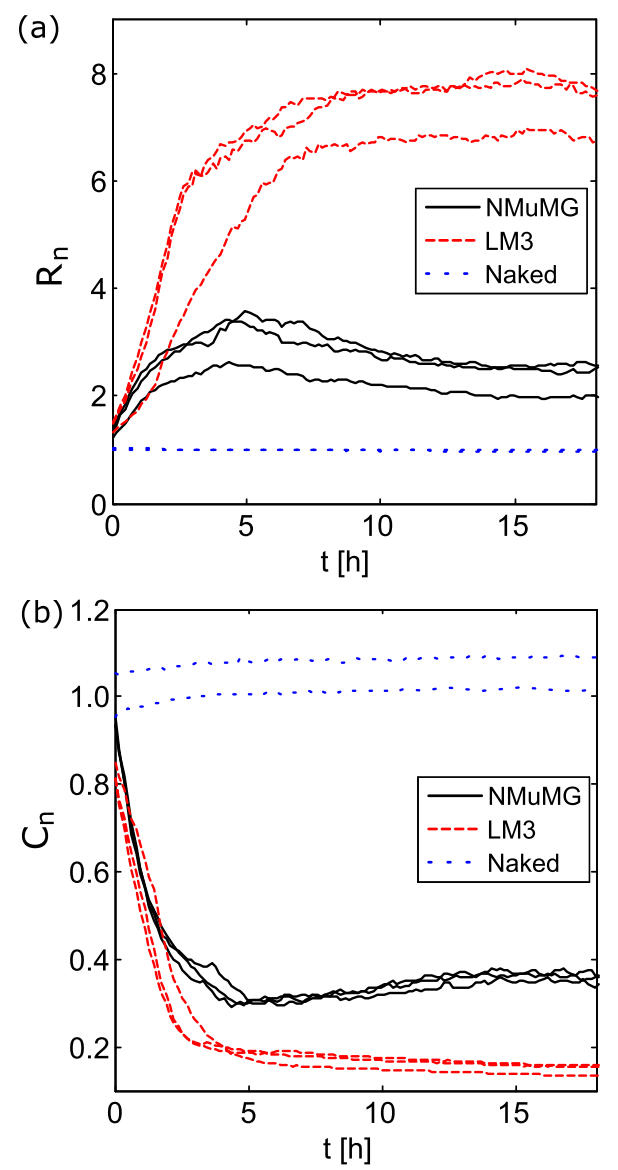

FIG. 3. Typical growth curves for normal (black solid lines) and cancerous (red dashed lines) cells. The dotted blue lines represent naked electrodes (control). At $t=0$, cells were seeded on different wells containing culture medium and impedance was measured as a function of time. The changes in normalized resistance (a) and capacitance (b) were registered at $4775 \mathrm{~Hz}$ and $100 \mathrm{kHz}$, respectively.

two wells were kept as control electrodes devoid of cells. When choosing the frequency to carry out these measurements, there is a trade-off between maximum sensitivity for cell growth monitoring and maximum discrimination. The experiments showed that at $4775 \mathrm{~Hz}$ the measurement is sensitive for both cell lines $\left(R_{n}\right.$ is greater than one and close to the maximum in the confluence phase; see Fig. 4). Simultaneously, at this frequency there is a big difference between both normalized resistance values, therefore making it useful to discriminate between normal and cancerous cells. The same criterion was used to select a frequency of $100 \mathrm{kHz}$ for capacitance measurements.

The results show that both cell lines increment the system's impedance until reaching a stationary state in approximately $13 \mathrm{~h}$ for NMuMG cells and $15 \mathrm{~h}$ for LM3 cells. This time was always longer for LM3 cells, and similar differences were previously observed and reported for other normal and cancerous cell lines [5]. Moreover, cancerous cells tend to grow faster (in terms of impedance) than normal cells, reaching much higher resistance (and much lower capacitance) values in the stationary state only a few hours later, and this behavior was observed in all the experiments carried out in this work.
These curves also show another characteristic of the normal cells, namely that they present a maximum resistance (and a minimum capacitance) approximately $5 \mathrm{~h}$ after the inoculation, after which the values decrease (increase in the case of capacitance) a little before reaching the stationary state. This phenomenon was observed in a previous work [5] for other normal cells and is adjudicated to the fact that they adhere to the culture surface very firmly at the beginning, but later they relax a little and start to move. Cancerous cells, however, have difficulties in adhering to the substrate and therefore don't reach the same initial adhesion strength and posterior relaxation.

Considering the particular behavior of normal cells previously described, we first analyzed the presence or absence of a normalized resistance peak during the first $15 \mathrm{~h}$ of this measurement phase. To this purpose, we applied a peakdetection algorithm to the normalized resistance curves and defined a parameter $R_{p}$, to which we assigned the value 1 if the peak was present and 0 otherwise. We also determined in each case the time $t_{p}$ in hours at which the peak occurred and, in the absence of this peak, we assigned to this parameter the maximum value of the time window, namely $t_{p}=15 \mathrm{~h}$.

In a previously published work [21], the authors defined two parameters that describe the adhesion and spreading of MDCK cells on microelectrodes used for ECIS measurements. One of these parameters is the half-time $t_{1 / 2}$, which was defined as the time required for the cells to spread out on half the available microelectrode area. To determine the capacitance value corresponding to this degree of coverage, the authors carried out computations by applying a theoretical model [Eqs. (2a)-(2c)]. For these computations, they used the values of some biological parameters (cell radius, distance between the basal membrane and the microelectrode, cell membrane capacitance) obtained by independent methods and reported in the literature. In this work, however, we studied two different cell lines and the values of the mentioned parameters were not yet available in the literature. Due to this lack of information, we decided to use a measure different to $t_{1 / 2}$ but with a similar physical meaning. We therefore defined the parameter $t_{G}$ as the time in hours required for the cells to spread out and reach a threshold normalized capacitance value of $C_{n}=0.4$, which was selected based on the characteristics of the growth curves.

The other parameter that was described in the aforementioned paper is the average slope of the capacitance shift, $s_{C}$, that represents the apparent rate of cell spreading and is given in $\left[h^{-1}\right]$. By following the same criterion explained in Ref. [21], we computed this value as the slope of a linear fit carried out between the representative values $C_{n}=0.5$ and $C_{n}=0.3$. The corresponding equation is

$$
C_{n}=-s_{C} t+b_{C},
$$

where $t$ is the time and $b_{C}$ is the $y$ intercept of the line. The slope of the line in Eq. (1) is negative, and therefore a minus sign appears to obtain positive values for $s_{C}$ (recall that this parameter represents the spread rate and therefore takes positive values). Four different features were thus obtained from the analysis of the growth phase, namely the resistance peak $R_{p}$ and the time of its occurrence $t_{p}$, the characteristic spreading time $t_{G}$ and the apparent rate of cell spreading $s_{C}$. 

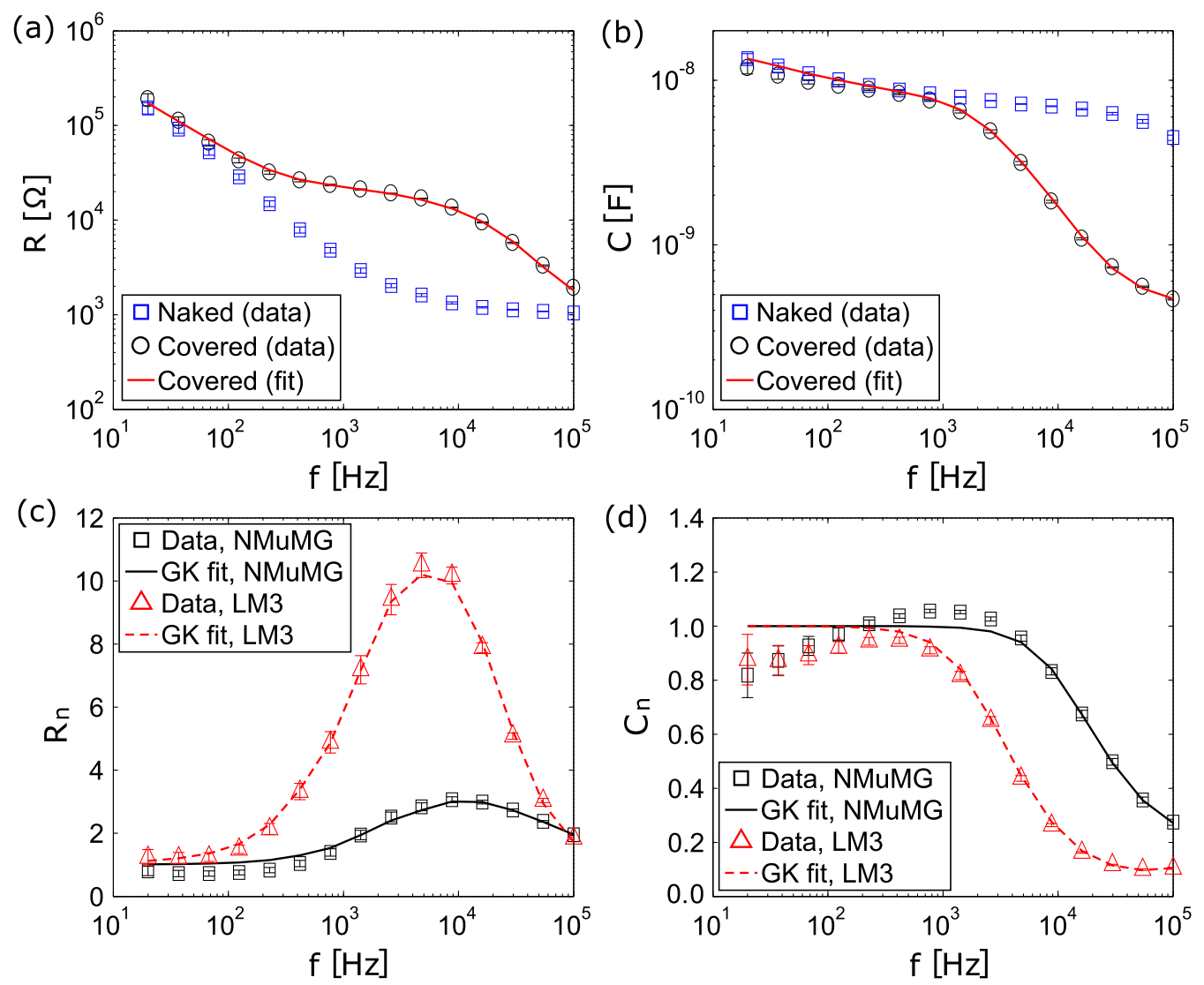

FIG. 4. Impedance spectra during the confluent phase. Figures (a) and (b) show experimental and fitted resistance and capacitance values, respectively, for naked (blue squares) and covered (black circles) electrodes with LM3 cells. Normalized resistance (c) and capacitance (d) are shown for both normal (black squares) and cancerous (red triangles) cells. The experimental values were fitted to the model by Giaever and Keese [Eqs. (2a)-(2c)] and the resulting theoretical normalized resistance and capacitance spectra are plotted as solid black lines for normal cells and dashed red lines for cancerous cells. The error bars represent standard deviation.

\section{B. Confluence phase}

The confluence phase starts when the impedance signals have reached a stationary state, which normally occurs between 10 and $16 \mathrm{~h}$ after seeding the cells. Thus, a growth phase of $18 \mathrm{~h}$ has proven to be sufficient to reach the stationary state in all our experiments. Typical LM3 and NMuMG impedance spectra are shown in Fig. 4. The results show significantly higher resistance values in the middle part of the spectrum and lower capacitance values at high frequencies for LM3 cells. This could be due to the LM3 cells forming stronger tight junctions than NMuMG cells, as this would result in a higher increase of the system's impedance [26]. Other possible explanation is that LM3 cells partially overlap, showing an impedance which is higher than the impedance of a monolayer.

We measured between 25 and 30 impedance spectra at a rate of 1 spectrum every $40 \mathrm{~min}$ to monitor the longtime fluctuations of the electrical properties of the confluent cell layers. From each set of measurements we obtained the following discrimination features: (1) average maximum normalized resistance $R_{n}$, which is related mainly to the distance between the basal membrane of the cells and the electrode surface and to the resistance between the cells [27], (2) average natural logarithm of the frequency (given in [Hz]) at which the maximum normalized resistance occurs $L_{f}$, (3) average minimum normalized capacitance $C_{n}$, which depends mainly on the cell membrane capacitance [27], (4) standard deviation of maximum normalized resistance $\sigma_{R}$, and (5) standard deviation of minimum normalized capacitance $\sigma_{C}$. Both $\sigma_{R}$ and $\sigma_{C}$ measure the fluctuations of the cell layer structure due to cell micromotion [27]. Note that all these quantities are dimensionless.

There are several mathematical models that describe the relationship between the impedance spectra during the confluent phase and some biological aspects of the cell cultures [26-28]. The simplest and most used of these models was introduced by Giaever and Keese [27] and is described by

$$
\begin{aligned}
& \frac{1}{Z_{\mathrm{cov}}(f)}=\frac{1}{Z_{\mathrm{nak}}(f)}\left[\frac{Z_{\mathrm{nak}}(f)}{Z_{\mathrm{nak}}(f)+Z_{m}(f)}\right. \\
& \left.+\frac{\frac{Z_{m}(f)}{Z_{\text {nak }}(f)+Z_{m}(f)}}{\frac{\gamma r_{c}}{2} \frac{I_{0}\left(\gamma r_{c}\right)}{I_{1}\left(\gamma r_{c}\right)}+R_{b}\left[\frac{1}{Z_{\text {nak }}(f)}+\frac{1}{Z_{m}(f)}\right]}\right], \\
& \alpha=\frac{\gamma r_{c}}{\sqrt{\frac{1}{Z_{\mathrm{nak}}(f)}+\frac{1}{Z_{m}(f)}}}, \\
& C_{m}=\frac{-j}{\pi f Z_{m}(f)} \text {. }
\end{aligned}
$$



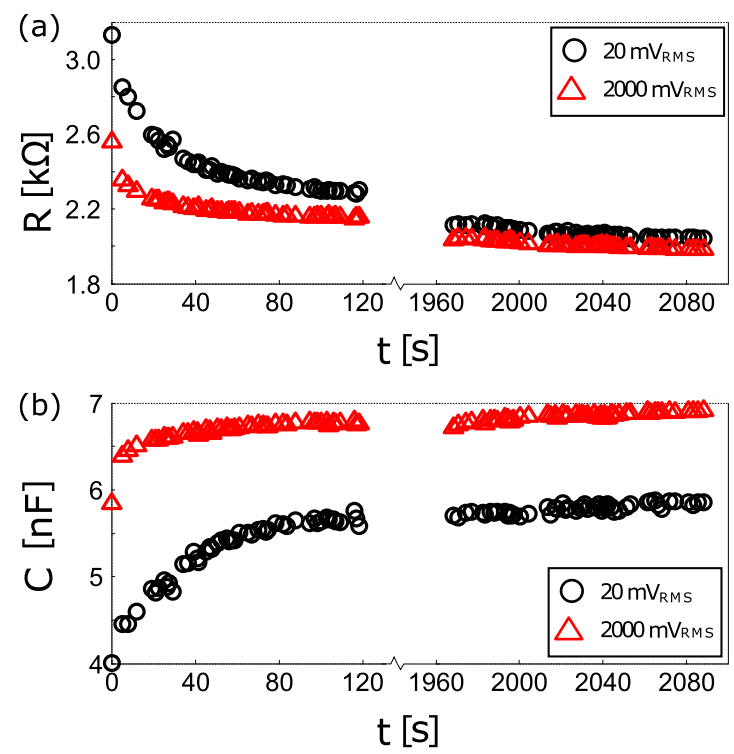

FIG. 5. Resistance (a) and capacitance (b) as a function of time during the wounding phase of a LM3 culture. The black circles and red triangles represent low- and high-voltage measurements, respectively. Each set of adjacent points represents a wounding cycle, as described in the text. For clarity purposes, only cycles 1 and 3 are shown.

Here $r_{c}$ is the cell radius and $\gamma=\sqrt{\rho / h}$, where $\rho$ is the electrical resistivity of the culture medium and $h$ is the distance between the basal membrane of the cells and the electrode surface. This model describes the relationship between the complex specific impedances of a cell-covered electrode $\left(Z_{\text {cov }}\right)$ and its corresponding naked electrode $\left(Z_{\text {nak }}\right)$. It has three parameters of biological relevance which we included in the set of discrimination features, namely: (1) constriction parameter $\alpha$ given in $\left[\Omega^{1 / 2} \mathrm{~cm}\right]$, which depends on the cell radius and the separation between the electrode surface and the cell layer, (2) intercellular joint resistance $R_{b}$ given in $\left[\Omega \mathrm{cm}^{2}\right]$, which measures the electrical resistance between adjacent cells, and (3) specific electrical cell membrane capacitance (considering both basal and apical membranes) $C_{m}$ given in $\left[\mu F / \mathrm{cm}^{2}\right]$. We computed the values of these parameters by fitting each experimental curve to the model and averaging over each data set.

\section{Wounding phase}

As described in Sec. II C 2, two signals were used to measure the impedance changes during the wounding phase, namely a high-voltage signal with an amplitude of $2 \mathrm{~V}_{\mathrm{RMS}}$ and a lowvoltage signal of $20 \mathrm{mV}_{\mathrm{RMS}}$, both with a frequency of $30 \mathrm{kHz}$. Figure 5 shows the time evolution of resistance and capacitance measurements during a typical wounding phase on LM3 cells using this protocol. As expected, a decrease in the system's resistance and a corresponding increase in its capacitance is observed as a consequence of the damage induced on the cell monolayer due to the application of the high-voltage signal. The results show that most of the impedance changes occur during the first wounding cycle, and this behavior was observed in all of the experiments carried out in this work. The changes in resistance and capacitance values during this cycle follow
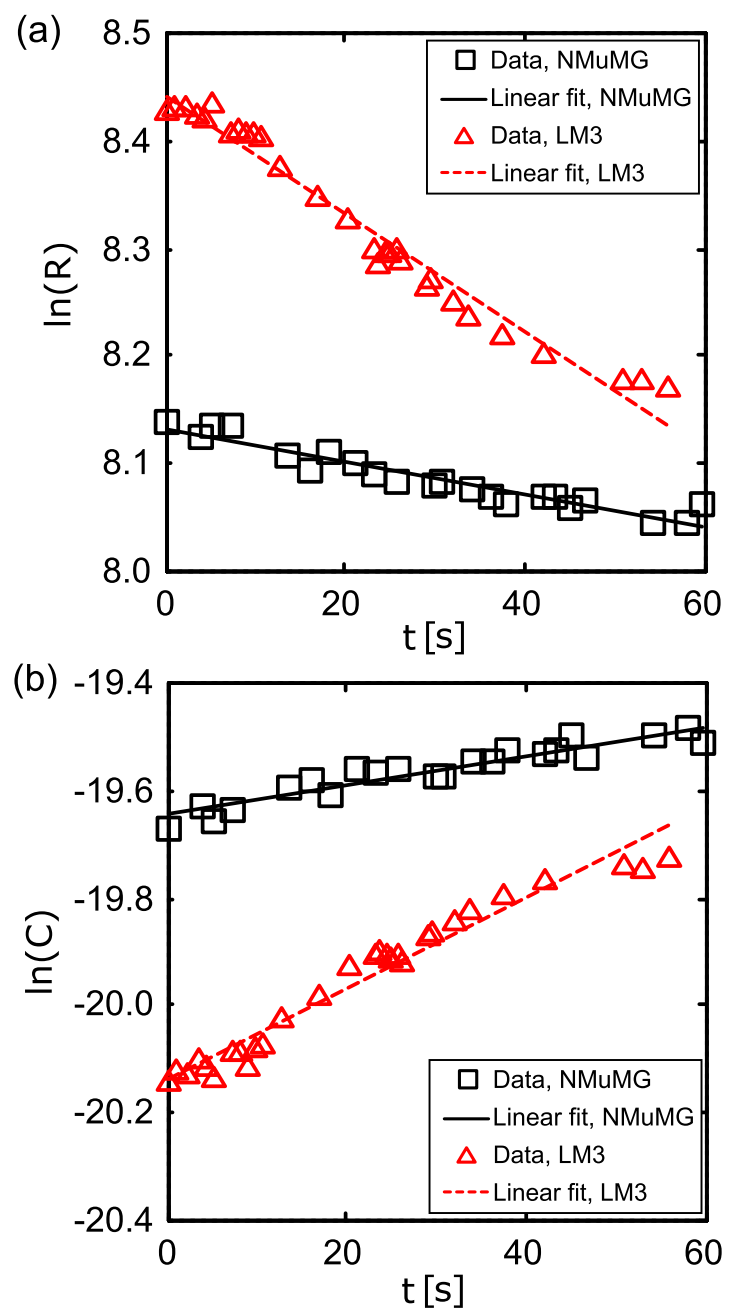

FIG. 6. Natural logarithm of resistance (a) and capacitance (b) as a function of time during the first wounding cycle for NMuMG and $\mathrm{LM} 3$ cultures. The values correspond to the control signal $\left(20 \mathrm{mV}_{\mathrm{RMS}}\right.$, $30 \mathrm{kHz}$ ). Normal cells are represented with black squares and solid lines, whereas red triangles and dashed lines represent cancerous cells. Linear fits were performed using Eqs. (3) and (4).

exponential-like curves, as shown in Figs. 6(a) and 6(b) for both cell types. We fitted these curves using the following models:

$$
\begin{aligned}
& \ln (R)=a_{R} t+b_{R}, \\
& \ln (C)=a_{C} t+b_{C} .
\end{aligned}
$$

Before taking the natural logarithm, $R$ was given in $[\Omega]$ and $\mathrm{C}$ in $[F]$. The slopes of these equations are given in $\left[\mathrm{s}^{-1}\right]$ and measure the apparent wounding rate, whereas the $y$ intercepts (dimensionless) are related to the values of $\mathrm{R}$ and $\mathrm{C}$ at $30 \mathrm{kHz}$ before applying the wounding signal, and they depend on the cell-substrate distance, the resistance between adjacent cells and the cell membrane capacitance. These four parameters were included in the set of discrimination features.

Previous results showed that the measured impedance changes as the applied voltage increases over some threshold [20] and the relationship between high- and low-voltage 
measurements was modeled as follows:

$$
\begin{aligned}
\frac{1}{\left|Z_{w}\right|} & =a_{1} \frac{1}{\left|Z_{m}\right|}+b_{1}, \\
\frac{1}{\theta_{w}} & =a_{2} \frac{1}{\theta_{m}}+b_{2} .
\end{aligned}
$$

Equation (5) describes an empirical relationship between impedance magnitudes measured with the wounding $\left(\left|Z_{w}\right|\right)$ and control $\left(\left|Z_{m}\right|\right)$ signals, while Eq. (6) describes the analogous relationship between impedance phase angles. They are related to the nonlinear behavior of the voltage-current relationship during cell wounding, as described in [20]. The slopes of both equations are dimensionless, while the $y$ intercepts are given in $\left[\Omega^{-1}\right]$ and $\left[\mathrm{rad}^{-1}\right]$ for Eqs. (5) and (6), respectively. These four empirical parameters were included in the set of discrimination features.

Finally, it was observed that the impedance changes during the wounding phase are more noticeable for cancerous cells than for normal cells. We therefore computed the fractional changes in resistance $\delta_{R}=\left(R_{\text {end }}-R_{0}\right) / R_{0}$ and capacitance $\delta_{C}=\left(C_{\text {end }}-C_{0}\right) / C_{0}$, where both quantities were measured with the control signal and $R_{\text {end }}$ and $C_{\text {end }}$ represent the last measured values before starting the healing phase. We included these dimensionless parameters in the set of discrimination features.

\section{Healing phase}

During this last measurement phase, the nondamaged cells that surround the electrode migrate and proliferate to repopulate the damaged portion of the monolayer [23]. Figure 7 shows an example of the time evolution in resistance and capacitance during the healing phase of both NMuMG and LM3 cultures. The measurements were carried out using the same frequencies as in the growth phase and had a duration of $8 \mathrm{~h}$.

As the wound heals, the normalized resistance increases and the normalized capacitance decreases, and the rates of change of these magnitudes are clearly different for both cell lines, as can be observed in Fig. 7. Therefore, we decided to use this information to find characteristic features in this phase. First, we computed the mean stationary normalized resistance and capacitance values by averaging the signals over the last 2 h (i.e., between $t=6 \mathrm{~h}$ and $t=8 \mathrm{~h}$ ). Let $t_{R}$ and $t_{C}$ be the times in hours at which the signals cross the mean values $\mathrm{R}_{\mathrm{s}}$ and $\mathrm{C}_{\mathrm{s}}$, respectively, for the first time. These parameters represent characteristic healing times and were included in the set of discrimination features. Finally, the following rates were computed:

$$
\begin{aligned}
& m_{R}=\frac{R_{s}-R_{0}}{t_{R}}, \\
& m_{C}=\frac{C_{0}-C_{s}}{t_{C}} .
\end{aligned}
$$

Here, $m_{R}$ and $m_{C}$ are positive quantities given in $\left[\mathrm{h}^{-1}\right]$ that represent the healing rates in terms of resistance and capacitance, respectively.
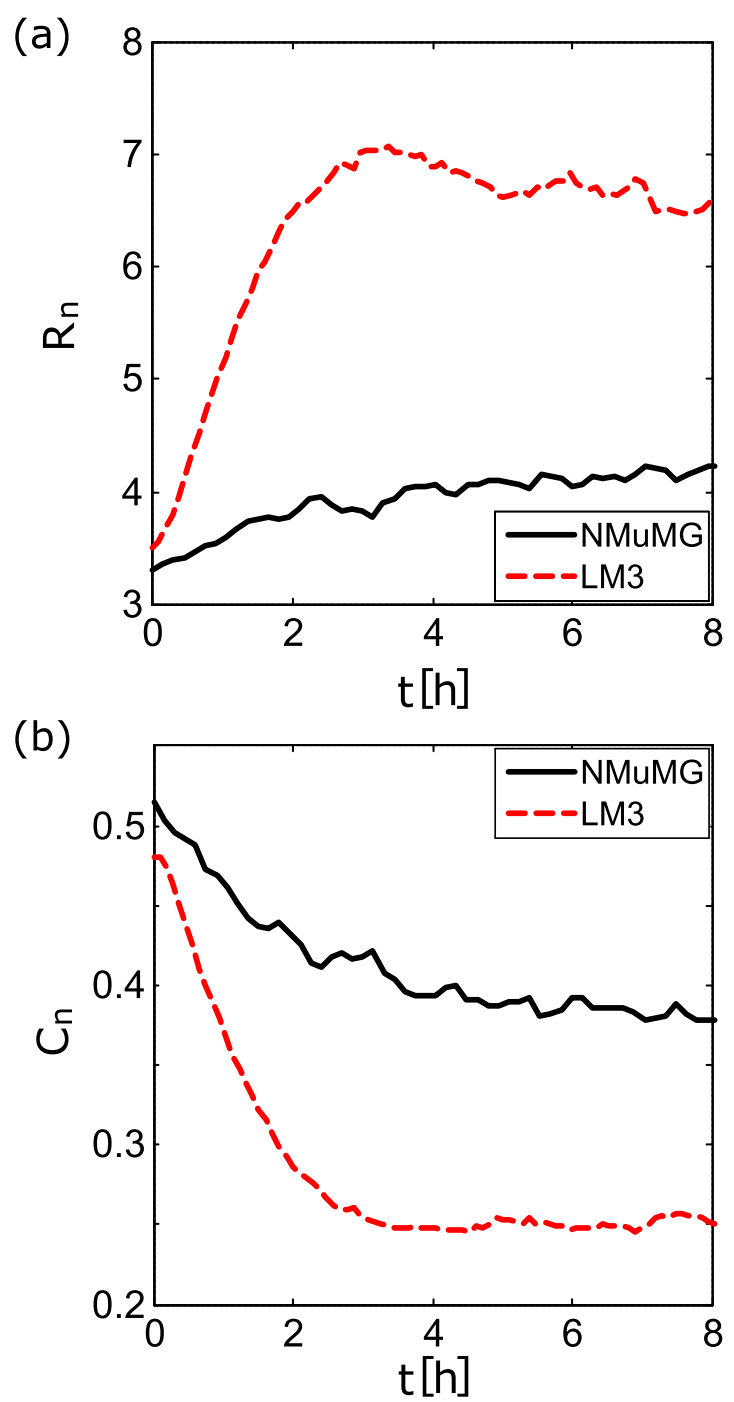

FIG. 7. Typical resistance and capacitance changes during the healing phase. At $t=0$ the wounding signal was interrupted and a noninvasive impedance measurement was started. As the nondamaged cells that surround the electrode start to migrate toward the wound and repopulate its surface, the measured resistance increases (a) and the capacitance decreases (b). The black solid lines correspond to a NMuMG culture, while the red dashed lines correspond to a LM3 culture. As expected, the healing rate (in terms of impedance) of cancerous cells resulted higher than for normal cells. Only values between $t=0$ and $t=8 \mathrm{~h}$ were considered in this analysis.

\section{DISCRIMINATION MODEL}

\section{A. Feature analysis and selection}

Table I summarizes the set of features obtained in this work, each of them presented within its corresponding measurement phase. The last three columns show the results of three statistical tests, namely the $F$ test, the Student's $t$ test and the Kolmogorov-Smirnov test [29]. The difference between the variances of the two data sets is analyzed through the $F$ test, whose null hypothesis states that their variances are actually consistent. The Student's $t$ test, on the other hand, checks whether the data sets have different means, and its null hypothesis states that their means are equal. In all cases, 
TABLE I. Discrimination features: mean values $\left(\mathrm{x}_{\mathrm{m}}\right)$, standard deviations $(\sigma)$, and statistical tests for $22 \mathrm{NMuMG}$ and 19 LM3 cell cultures. The values are given in the units described in the text for each feature. In the last three columns, the values represent the probabilities under each test that the data sets corresponding to normal and cancerous cells come from the same distribution. Values less than $1 \times 10^{-6}$ have been rounded down to 0 . Features marked with $(*)$ are considered as relevant in our discrimination model. G, Growth; C, Confluence; W, Wounding; H, Healing.

\begin{tabular}{|c|c|c|c|c|c|c|c|c|}
\hline \multirow[b]{2}{*}{ Phase } & \multirow[b]{2}{*}{ Feature } & \multicolumn{2}{|c|}{ NMuMG } & \multicolumn{2}{|c|}{ LM3 } & \multirow[b]{2}{*}{$F$ test } & \multirow[b]{2}{*}{$t$ test } & \multirow[b]{2}{*}{$\mathrm{KS}$ test } \\
\hline & & $x_{m}$ & $\sigma$ & $x_{m}$ & $\sigma$ & & & \\
\hline \multirow{4}{*}{ G } & $t_{p}(*)$ & 8.5 & 4.1 & 13.6 & 1.9 & $2.1 \times 10^{-3}$ & $1.0 \times 10^{-5}$ & $9.7 \times 10^{-4}$ \\
\hline & $R_{p}(*)$ & 0.95 & 0.21 & 0.52 & 0.51 & $2.2 \times 10^{-4}$ & $2.4 \times 10^{-3}$ & 0 \\
\hline & $t_{G}$ & 9.1 & 6.1 & 5.3 & 4.3 & $1.4 \times 10^{-1}$ & $2.6 \times 10^{-2}$ & $2.6 \times 10^{-4}$ \\
\hline & $s_{C}(*)$ & $4.1 \times 10^{-2}$ & $3.4 \times 10^{-2}$ & 0.12 & $5.7 \times 10^{-2}$ & $2.7 \times 10^{-2}$ & $1.2 \times 10^{-5}$ & $2.1 \times 10^{-4}$ \\
\hline \multirow{8}{*}{$\mathrm{C}$} & $R_{n}(*)$ & 2.8 & 0.66 & 7.0 & 2.0 & $2.1 \times 10^{-5}$ & 0 & 0 \\
\hline & $L_{f}(*)$ & 9.6 & 0.52 & 8.6 & 0.20 & $1.4 \times 10^{-2}$ & $1.4 \times 10^{-6}$ & $6.0 \times 10^{-6}$ \\
\hline & $C_{n}(*)$ & 0.30 & $7.9 \times 10^{-2}$ & 0.17 & $4.1 \times 10^{-2}$ & $5.8 \times 10^{-4}$ & 0 & 0 \\
\hline & $\alpha(*)$ & 2.6 & 0.66 & 5.7 & 0.93 & 0.21 & 0 & 0 \\
\hline & $R_{b}(*)$ & 0.64 & 0.42 & 3.2 & 1.9 & 0 & $8.8 \times 10^{-5}$ & $2.9 \times 10^{-5}$ \\
\hline & $C_{m}$ & 2.0 & 0.92 & 2.3 & 0.42 & $3.5 \times 10^{-3}$ & 0.23 & 0.74 \\
\hline & $\sigma_{R}$ & 0.35 & 0.24 & 1.2 & 0.81 & $4.9 \times 10^{-6}$ & $5.0 \times 10^{-4}$ & $1.0 \times 10^{-3}$ \\
\hline & $\sigma_{C}^{K}$ & $5.1 \times 10^{-2}$ & $3.6 \times 10^{-2}$ & $2.0 \times 10^{-2}$ & $1.8 \times 10^{-2}$ & $7.6 \times 10^{-3}$ & $2.7 \times 10^{-3}$ & $1.1 \times 10^{-2}$ \\
\hline \multirow{10}{*}{ W } & $a_{1}$ & 0.61 & 0.26 & 0.68 & 0.16 & 0.20 & 0.43 & 1.0 \\
\hline & $b_{1}$ & $2.0 \times 10^{-4}$ & $1.6 \times 10^{-4}$ & $1.0 \times 10^{-4}$ & $4.4 \times 10^{-5}$ & $2.4 \times 10^{-4}$ & $6.0 \times 10^{-2}$ & 0.31 \\
\hline & $a_{2}$ & 0.51 & 0.44 & 0.55 & 0.22 & $4.2 \times 10^{-2}$ & 0.85 & 0.92 \\
\hline & $b_{2}$ & -1.3 & 1.1 & -1.1 & 0.42 & $5.8 \times 10^{-3}$ & 0.45 & 1.0 \\
\hline & $a_{R}$ & $-2.0 \times 10^{-3}$ & $2.2 \times 10^{-3}$ & $-4.6 \times 10^{-3}$ & $7.7 \times 10^{-4}$ & $1.9 \times 10^{-3}$ & $1.4 \times 10^{-3}$ & $8.6 \times 10^{-4}$ \\
\hline & $b_{R}(*)$ & 7.8 & 0.33 & 8.5 & 0.16 & $2.6 \times 10^{-2}$ & $1.9 \times 10^{-6}$ & $2.5 \times 10^{-6}$ \\
\hline & $a_{C}(*)$ & $2.5 \times 10^{-3}$ & $2.9 \times 10^{-3}$ & $8.2 \times 10^{-3}$ & $1.4 \times 10^{-3}$ & $3.4 \times 10^{-2}$ & $5.8 \times 10^{-6}$ & $8.6 \times 10^{-4}$ \\
\hline & $b_{C}(*)$ & -19.4 & 0.31 & -20.4 & 0.29 & 0.83 & 0 & $2.7 \times 10^{-5}$ \\
\hline & $\delta_{R}(*)$ & -0.20 & 0.15 & -0.51 & $4.9 \times 10^{-2}$ & $1.2 \times 10^{-3}$ & $5.2 \times 10^{-6}$ & $2.5 \times 10^{-6}$ \\
\hline & $\delta_{C}(*)$ & 0.30 & 0.30 & 1.7 & 0.56 & $3.7 \times 10^{-2}$ & $2.6 \times 10^{-6}$ & $2.5 \times 10^{-6}$ \\
\hline \multirow{4}{*}{$\mathrm{H}$} & $t_{R}$ & 4.5 & 2.3 & 4.5 & 1.7 & 0.26 & 1.0 & 0.9 \\
\hline & $m_{R}(*)$ & $5.3 \times 10^{-2}$ & $4.6 \times 10^{-1}$ & 1.4 & 0.966 & $3.8 \times 10^{-3}$ & $1.4 \times 10^{-5}$ & 0 \\
\hline & $t_{C}$ & 4.6 & 1.9 & 4.9 & 1.3 & 0.29 & 0.67 & 0.88 \\
\hline & $m_{C}(*)$ & $5.1 \times 10^{-2}$ & $7.8 \times 10^{-2}$ & 0.46 & 0.19 & $7.0 \times 10^{-4}$ & 0 & 0 \\
\hline
\end{tabular}

we used the unequal-variance $t$ test. Finally, the KolmogorovSmirnov test tests the null hypothesis that the two data sets are drawn from the same distribution. For each feature, the value presented in each of these columns indicates the probability under the corresponding test that the related null hypothesis is true. Therefore, low probabilities suggest that the two populations are different, and generally a value $p<0.05$ is considered as statistically significant.

By looking at the results presented on Table I, it can be seen that, for example, the feature $R_{n}$ has a very high discriminant capacity. We could indeed use this single feature to classify an unknown sample as being normal or cancerous. However, these results are specific of NMuMG and LM3 cells, and it can be possible that these differences are not as significant for other cell types. Other features, however, could be less specific and therefore represent a more universal behavior of normal and cancerous cells. This work is aimed to obtain a general method that could be extended to other cell types present in different tissues. The use of a multidimensional feature vector as the input for a classification algorithm has the advantage of being more general in the sense that it takes into account different aspects of the cell behavior.
Before using any discrimination algorithm, a dimensionality reduction based on feature selection results of practical importance [30] and gives a better insight on the problem by focusing only on those features that give a significant separation between both populations. We considered values of $p<1 \times 10^{-3}$ as most significant and selected only those features that presented this condition simultaneously under the $\mathrm{KS}$ test and one of the other tests. Following this criterion, the results presented in Table I suggest that only 15 of the initial 26 features are relevant in terms of their discriminant capacity. Hence, the analysis that follows is based on this 15-dimensional feature vector.

\section{B. Linear discriminant analysis (LDA)}

The purpose of the LDA algorithm is to find a unit vector $\mathbf{w}$ that maximizes the ratio of between-class to within-class scatter when the data points are projected onto it, thus giving a maximum separation between classes [30]. Let $\mathbf{x}$ be an unclassified data point. The euclidean distances from this point to both population means along the direction given by $\mathbf{w}$ are 


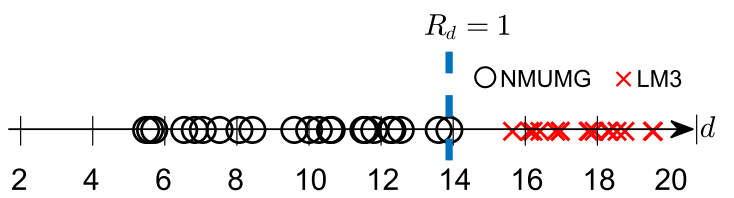

FIG. 8. Euclidean distances along the direction given by the discriminant vector w. Data points are represented as black circles and red crosses for normal and cancerous cells, respectively. The dashed blue line located at $d \simeq 13.9$ represents the boundary between both populations, i.e., a hyperplane where the euclidean distances to both population means are the same and therefore $R_{d}=1$. The model classifies a new data point as normal if its projection along $\mathbf{w}$ lays at the left side of this boundary $\left(R_{d}>1\right)$, and as cancerous if it lays at the right side of the boundary $\left(R_{d}<1\right)$.

given by

$$
\begin{aligned}
& d_{N}=\mathbf{w}^{T} \cdot \mathbf{x}-\mu_{N}, \\
& d_{L}=\mathbf{w}^{T} \cdot \mathbf{x}-\mu_{L},
\end{aligned}
$$

where $d_{N}$ and $\mu_{N}$ are the euclidean distance and population mean (projected onto w) corresponding to NMuMG cells, and $d_{L}$ and $\mu_{L}$ correspond to LM3 cells. If we consider these distances as new features, the problem is reduced to one dimension. The criterion to choose whether an unknown feature vector $\mathbf{x}$ comes from a normal or cancerous cell culture is to measure the ratio between euclidean distances $R_{d}=d_{L} / d_{N}$ and to assume that values of $R_{d}$ less than 1 correspond to cancerous cells and values greater than 1 represent normal cells.

Before applying this algorithm to our data, a consideration must be taken into account. The sets of normal and cancerous cells' experimental data collected in this work consist of both complete and incomplete feature vectors. Thus, a method for treating missing data is to be considered before carrying out the LDA. Following the recommendations given by Hufnagel [31], we determined the average proportion of missing observations in our experiments, which was $m_{N}=0.20$ for NMuMG cells and $m_{L}=0.26$ for LM3 cells. The size of the initial samples was 22 for normal and 19 for cancerous cells. Based on this information, the most reasonable option for completing the missing observations seemed to be Dear's method [32,33], which is based on an approximation to the first principal component and makes no assumptions about the distributions of the missing features. We therefore carried out the LDA using the set of complete feature vectors after applying Dear's method to fill in the missing values.

Figure 8 shows the projections of the 15-dimensional feature vectors on the direction given by the discriminant unit vector $\mathbf{w}$. The vertical line represents the hyperplane perpendicular to $\mathbf{w}$ for which $R_{d}=1$ and the experimental data points corresponding to both cell lines are scattered around it. As it can be observed, a clear separation between populations is achieved and only one data point corresponding to NMuMG cells is misclassified, yielding $4.5 \%$ of false positives and no false negatives.

\section{CONCLUSIONS}

We have proposed a methodology which unifies different spectral impedance measurement techniques to obtain relevant characteristics of normal and cancerous cell cultures. The measurement protocol was divided in four phases based on different aspects of the cell cultures. We used the resulting characteristic features to discriminate between both cell types by means of a linear discriminant analysis. The method has proven effective and presented only $4.5 \%$ of false positives and no false negatives for a total of $22 \mathrm{NMuMG}$ and 19 LM3 in vitro cell cultures. Applied to an unknown sample, the assay returns a number which has the potential of being used as an indicator on cancer diagnostic procedures.

The analysis could easily be extended to other cell lines to investigate whether the differences observed between normal and cancerous glandular cells are also present in other tissues. The results obtained in this work are in concordance with previous studies carried out on fibroblasts. Particularly, the normal cell line NMuMG presented a resistance peak during the growth phase [5] and the cancerous cell line LM3 showed higher resistance and lower capacitance than the normal one [27].

The discrimination method described in this paper is relatively simple in the sense that both the measurement procedure and the data processing are fully automated, although its application on cancer diagnosis would require samples that can in reality be obtained only by invasive means. Nevertheless, in the vast majority of cases a definite diagnosis of a malignant tumor requires the analysis of a tissue sample, and the assay described in this work could be carried out on these samples. Moreover, research could be conducted on this basis to evaluate the possibility of performing a similar assay on in vivo samples.

We consider that these results can be a starting point to understand the differences in the electrical behavior of normal and cancerous cells cultured in vitro during the whole measurement interval. The evaluation of more complex models from which the characteristic features are extracted in each phase could help enhance their discriminant capacity. Nevertheless, the technique has proven to be effective and our next objective is to implement it to determine the presence of cancerous cells in cocultures consisting of a controlled mix of both cell types in a single well containing a set of spatially distributed microelectrodes.

\section{ACKNOWLEDGMENTS}

We thank Dr. Soledad Bustillo (Laboratorio de Cultivos Celulares, Universidad Nacional del Nordeste, Corrientes, Argentina) for generously providing us the cell lines used in this paper, Dr. Willy Pregliasco (Grupo de Física Forense, Centro Atómico Bariloche, San Carlos de Bariloche, Argentina) for his advice and recommendations regarding the statistical aspects of the work, and Pablo Vallina for his support on the construction of the measurement platform. F.E.G. acknowledges a scholarship from Comisión Nacional de Investigaciones Científicas y Técnicas (CONICET), Argentina. 
[1] C. A. Almeida, and S. A. Barry, Cancer: Basic Science and Clinical Aspects (Wiley-Blackwell, London, 2010), Chap. 6, pp. 115-134.

[2] R. A. Smith, V. Cokkinides and O. Brawley, CA cancer. J. Clin. 59, 27 (2009).

[3] C. Oliveira and A. Saraiva, Radiol. Bras. 43, 229 (2010).

[4] H. D. Nelson, E. S. O’Meara, K. K. Kerlikowske, S. Balch and D. Miglioretti, Evidence summary: False-positive and false-negative rates of digital mammography screening, https://www.uspreventiveservicestaskforce.org/Page/Docu ment/evidence-summary-false-positive-and-false-negativerates-of-/breast-cancer-screening1.

[5] I. Giaever and C. R. Keese, IEEE Trans. Biomed. Eng. 33, 242 (1986).

[6] E. Zudaire, N. Cuesta, V. Murty, K. Woodson, L. Adams, N. Gonzalez, A. Martínez, G. Narayan, I. Kirsch, W. Franklin, F. Hirsch, M. Birrer, and F. Cuttitta, J. Clin. Invest. 118(2), 640 (2008).

[7] G. Park, C. K. Choi, A. E. English, and T. E. Sparer, Cell Biol. Int. 33, 429 (2009).

[8] Q. Liu, J. Yu, L. Xiao, J. C. O. Tang, Y. Zhang, P. Wang, and M. Yang, Biosens. Bioelectron. 24, 1305 (2009).

[9] L. R. Arias, C. A. Perry, and L. Yand, Biosens. Bioelectron. 25, 2225 (2010).

[10] J. Hong, K. Kandasamy, M. Marimuthu, C. S. Choi, and S. Kim, Analyst 136, 237 (2011).

[11] B. Blad and B. Baldetorp, Physiol. Meas. 17, A105 (1996).

[12] B. H. Brown, J. A. Tidy, K. Boston, A. D. Blackett, R. H. Smallwood, and F. Sharp, Lancet 355, 892 (2000).

[13] B. A. Wilkinson, R. H. Smallwook, A. Keshtar, J. A. Lee, and F. Hamdy, J. Urol. 168, 1563 (2002).

[14] P. Åberg, I. Nicander, J. Hansson, P. Geladi, U. Holmgren, and S. Ollmar, IEEE Trans. Biomed. Eng. 51, 2097 (2004).

[15] D. C. Lovelady, T. C. Richmond, A. N. Maggi, C. M. Lo, and D. A. Rabson, Phys. Rev. E 76, 041908 (2007).

[16] R. B. Owens, J. Natl. Cancer. Inst. 52, 1375 (1974).

[17] A. Urtreger, V. Ladeda, L. Puricelli, A. Rivelli, M. Vidal, E. Delustig, and E. Joffe, Int. J. Oncol. 11(3), 489 (1997).
[18] Applied Biophysics, Improving experiment repeatability with cysteine, http://www.biophysics.com/publications/Improving Experiment Repeatability with Cysteine.pdf.

[19] F. E. Giana, F. J. Bonetto, and M. I. Bellotti, in Proceedings of the 2017 IEEE 8th Latin American Symposium on Circuits Systems (LASCAS'17), Bariloche (IEEE, New York, 2017), pp. 1-4.

[20] M. I. Bellotti, F. E. Giana, and F. J. Bonetto, Meas. Sci. Technol. 26, 085701 (2015).

[21] J. Wegener, C. R. Keese, and I. Giaever, Exp. Cell Res. 259, 158 (2000).

[22] Z. Környei, A. Czirók, T. Vicsek, and E. Madarász, J. Neurosci. Res. 61, 421 (2000).

[23] C. R. Keese, J. Wegener, S. R. Walker, and G. I., Proc. Natl. Acad. Sci. USA 101, 1554 (2004).

[24] N. Mondal, D. Mondal, C. RoyChaudhuri, A. Barui, S. Dhara, and J. Chatterjee, in Proceedings of the 2011 IEEE/NIH 5th Life Science Sistems and Applications Workshop (LiSSA'11), Bethesda (IEEE, New York, 2011), pp. 47-50.

[25] R. Riahi, Y. Yang, D. D. Zhang, and P. K. Wong, J. Lab. Autom. 17, 59 (2012).

[26] C. M. Lo, C. R. Keese, and I. Giaever, Biophys. J. 69, 2800 (1995).

[27] I. Giaever and C. R. Keese, Proc. Natl. Acad. Sci. USA 88, 7896 (1991).

[28] E. Urdapilleta, M. Bellotti, and F. J. Bonetto, Phys. Rev. E 74, 041908 (2006).

[29] W. H. Press, S. A. Teukolsky, W. T. Vetterling, and B. P. Flannery, Numerical Recipes in C: The Art of Scientific Computing, 3rd ed. (Cambridge University Press, Cambridge, 2007), pp. 726-740.

[30] S. Marsland, Machine Learning: An Algorithmic Perspective, (Chapman \& Hall/CRC, London, 2009), Chap. 10, pp. 221-226.

[31] G. Hufnagel, Biom. J. 30, 69 (1988).

[32] R. E. Dear, A Principal Component Missing Data Method for Multiple Regression Models. Technical report SP-86, (Systems Development Corporation, Santa Monica, CA, 1959).

[33] P. H. Rossi, J. D. Wright, and A. B. Anderson, Handbook of Survey Research (Academic Press, San Diego, 1983), pp. 442448. 